\title{
Motor harmonic current detection system based on magnetic sensor
}

\author{
Jian Jiang ${ }^{1, a}$, Zhongming Pan $^{1, b}$, Han Zhou $^{1, c}$ \\ ${ }^{1}$ College of Mechatronics Engineering and Automation, National University of Defense \\ Technology, Changsha 410073, Hunan, China. \\ a13107210194@163.com, bzhongmingpan@163.com, czhounng2@163.com
}

\begin{abstract}
Keywords: Harmonic detection; Magnetic sensor; Window functions; Interpolation algorithm; Fast Fourier Transform
\end{abstract}

\begin{abstract}
In order to obtain the motor current signal conveniently and solve the problem caused by the fundamental frequency fluctuation of the power network, a current harmonic detection system based on magnetic sensor is proposed in this paper. This system will simplify the measurement process, as it uses the magnetic sensor instead of the traditional current transformer to obtain harmonic current waveform. In order to improve the calculation accuracy, the window function and interpolation algorithm is used in the detection system to correct the result calculated by Fast Fourier Transform, which can depression the disturbance of asynchronous sampling and non-integral period truncation. Test results show that the measurement error of frequency and the relative error of amplitude is small,which can prove that the proposed system is simple and effective.
\end{abstract}

\section{Introduction}

When the motor is in fault, the high harmonic components contained in the power line current will be changed, which can provide a reference for the motor fault diagnosis by detecting the change. The key question is how to measure the harmonic component of the electric current of the motor accurately and quickly.

In order to measure the harmonic components of power line current, first we need to obtain the electric current signal, and then use the appropriate method to estimate the harmonic components of the current signal. At present, the current transformer is a common device $[1,2,3]$ used to measure power line current signal. In order to convert the larger current signal into weak current signal,which is easily to be measured ,the current harmonic detection system need to wind the power line to the transformer. However, motors are often work in complex environment, the power line is usually thick and the external often cover a protective layer, the use of transformers will often give the motor power line current signal detection inconvenience. For harmonic detection algorithm [4,5], the most widely used method is based on FFT. The high accuracy estimation by this method needs the condition of synchronous sampling,however the fundamental frequency of the motor can fluctuate around the rated frequency which can cause asynchronous sampling and bring influence to the accuracy detection .

In order to obtain the motor current signal and solve the problem of the fundamental frequency fluctuation of the power network, this paper designs a power line current harmonic detection system based on giant magneto impedance (GMR) magnetic sensor. It uses hanning window interpolation FFT algorithm to analyze the harmonic wave signal, which can effectively solve the problem of accuracy reduction of the FFT harmonic detection algorithm under an asynchronous sampling condition. The final experimental results show that the AC magnetic field signal generated by the electric current can be detected by GMR magnetic sensor with any change to the motor power line, and the window interpolation FFT algorithm can be used to estimate the harmonic components of the motor even under an asynchronous sampling condition. 


\section{Window interpolation algorithm}

Because of the fluctuation of the fundamental frequency of the power network, the non synchronous sampling will appear, and if traditional FFT algorithm was used to analyze the power line current signal, the Zou Bo effect and fence effect[10] would be in the results and affect the accuracy. In order to reduce the fence effect in FFT algorithm, a high accuracy harmonic detection method based on FFT calculation results is proposed by V.K.Jain[11] et al., and a window is used to suppress the Zou Bo effect and improve the accuracy of harmonic detection by T.Grandke[12]. This method, which is based on the suppression of the Zou Bo effect by the window and interpolation, is called the window interpolation FFT algorithm. At present, the research and application on the window interpolation algorithm are too numerous to count [6-9]. Based on the actual situation of the power line, this paper compares several common window functions, and finally selects the interpolation FFT algorithm with Hanning window to estimate (or detect) the electric current generated by the power line current.

When the FFT harmonic analysis is carried out, the main lobe width and the side lobe amplitude of the amplitude spectrum of the window function are narrow, so that the spectrum leakage and the wrinkling phenomenon of the FFT spectrum are reduced. However, so far there is no such window function amplitude spectrum can achieve the main valve width is narrow, while the side lobe amplitude and small ideal condition. Therefore, to select the best window function should accord to the actual application needs. This paper mainly studies how to use FFT algorithm to detect the harmonic components of the power line current signal, the frequency of which is integer multiple of the fundamental frequency (about $50 \mathrm{~Hz}$ ), the frequency interval is big, it is supposed to select a window with wide main lobe width and low side lobe.But noise is another aspect needed to be considered, the wider the main lobe width of the window is ,the bigger the influence to the detecting accuracy caused by spectrum of noise will be. Finally, Hanning is chosed as it has a relative narrow main lobe and a relative low side lobe.

\section{Harmonic detection system based on Giant Magneto Impedance magnetic sensor}

\subsection{System introduction}

Fig. 1 gives a block diagram of the harmonic detection system based on magnetic sensor. The system is composed of three parts, magnetic sensor, acquisition card (USB2852) and PC. Among them, magnetic sensor including magnetic probe (HMC1021), power supply circuit, signal conditioning circuit, reset circuit, anti aliasing filter circuit.

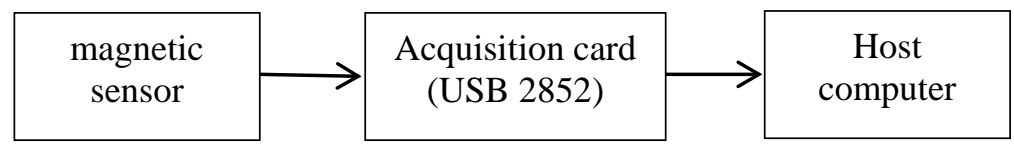

Fig.1 The block diagram of the harmonic detection system

The work process of the harmonic detection system is as follows: magnetic sensor (see Fig.2a) is used to detect the magnetic field generated by the magnetic probe into voltage signal, and signal conditioning circuit to amplify and filter the voltage signal, and then output to the acquisition card; acquisition card (real see Fig.3b) on the magnetic sensor output signal A/D conversion to digital signal, the host computer to read the digital signal in the acquisition card, then use software to estimate the harmonic components of this signal. 


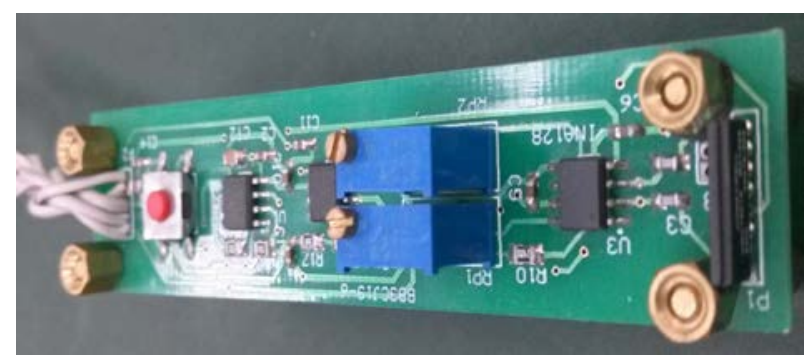

(a) Magnetic sensor

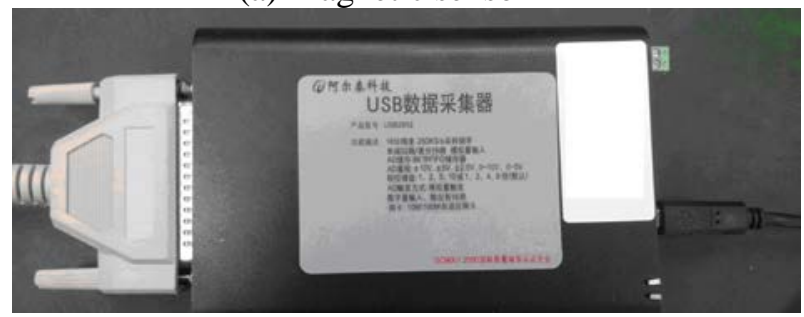

(b) Acquisition card

Fig.2 The hardware diagram

\subsection{Magnetic sensor circuit}

Sensing technology and signal conditioning technology are collectively referred to the detection technology, the key to achieve satisfactory accuracy and requirements of the measurement [13]. Selecting the appropriate sensitive components, and designing a suitable analog front end according to the characteristics of sensitive components and the environment is the first and focus issue on the design of the detection system.

In order to truly respond to the size of the current in the electric wire and fast track of the change of the current, the magnetic probe should have the advantages of high linearity, low hysteresis and high sensitivity. Table 1 lists several magnetic probes with good performance. Because the hysteresis error and nonlinearity can affect the magnetic probe output voltage and the measured current waveform similarity, so in order to be able to accurately measure the harmonic components of the current waveform, magnetic probe should be selected between HMC1051 and HMC1021. Considering the sensitivity and linear range of the magnetic probe, the HMC1021 is chosen as the magnetic probe of the harmonic detection system, and the according analog front end circuit is designed(see fig.4).

Table 1 Magnetic probe commonly used abroad

\begin{tabular}{cccccc}
\hline $\begin{array}{c}\text { Production } \\
\text { company }\end{array}$ & Model & $\begin{array}{c}\text { Linear range } \\
(\mathrm{Oe})\end{array}$ & $\begin{array}{c}\text { Sensitivity } \\
(\mathrm{mV} / \mathrm{O})\end{array}$ & $\begin{array}{c}\text { Nonlinearity } \\
(\% \mathrm{FS})\end{array}$ & $\begin{array}{c}\text { Hysteresis } \\
\text { error(\%FS) }\end{array}$ \\
\hline \multirow{2}{*}{ NVE } & AA003-02 & $2.0 \sim 14$ & $2.0 \sim 3.2$ & 2 & 4 \\
& AA005-02 & $10 \sim 70$ & $0.45 \sim 0.65$ & 2 & 4 \\
Honey-well & HMC1021 & $-6.0 \sim+6.0$ & $0.8 \sim 1.2$ & 1.6 & 0.05 \\
Spinic & HMC1051 & $-6.0 \sim+6.0$ & $0.8 \sim 1.2$ & 1.8 & 0.06 \\
\end{tabular}

The HMC1021 is bipolar according to its input and output, thus can be directly used to measure the alternating magnetic field, with no external bias magnetic field. But in a strong magnetic environment of more than 10 Gauss, the sensor can be remagnetized, resulting in low sensitivity and accuracy. As shown in Fig.4 , set / reset circuit is formed by the chip IRF7105 including 2 enhanced MOS tubes, and the gates of the 2 MOS tubes are connected to a I/O port (be expressed as SR_IN)of the acquisition card(USB2852). Only before the beginning of the measurement, USB2852 set a $5 \mu$ s negative pulse to SR_IN port, otherwise SR_IN maintains high level. When the voltage of SR_IN is from positive to negative, the P MOS tube will be conducted and the N one blocked ,thus charge in C4 will discharge through the setting resistor and C3 (set). When the voltage of SR_IN is from negative to positive ,the $\mathrm{P}$ MOS tube will be blocked and the $\mathrm{N}$ one conducted ,thus charge in C3 will discharge through the setting resistor and N MOS tube(reset), At the same time, Power charges C4 through resistor R11. By setting or resetting, the sensor can be recovered from the strong magnetic environment to the original state of magnetization, and 
maintains the maximum sensitivity.

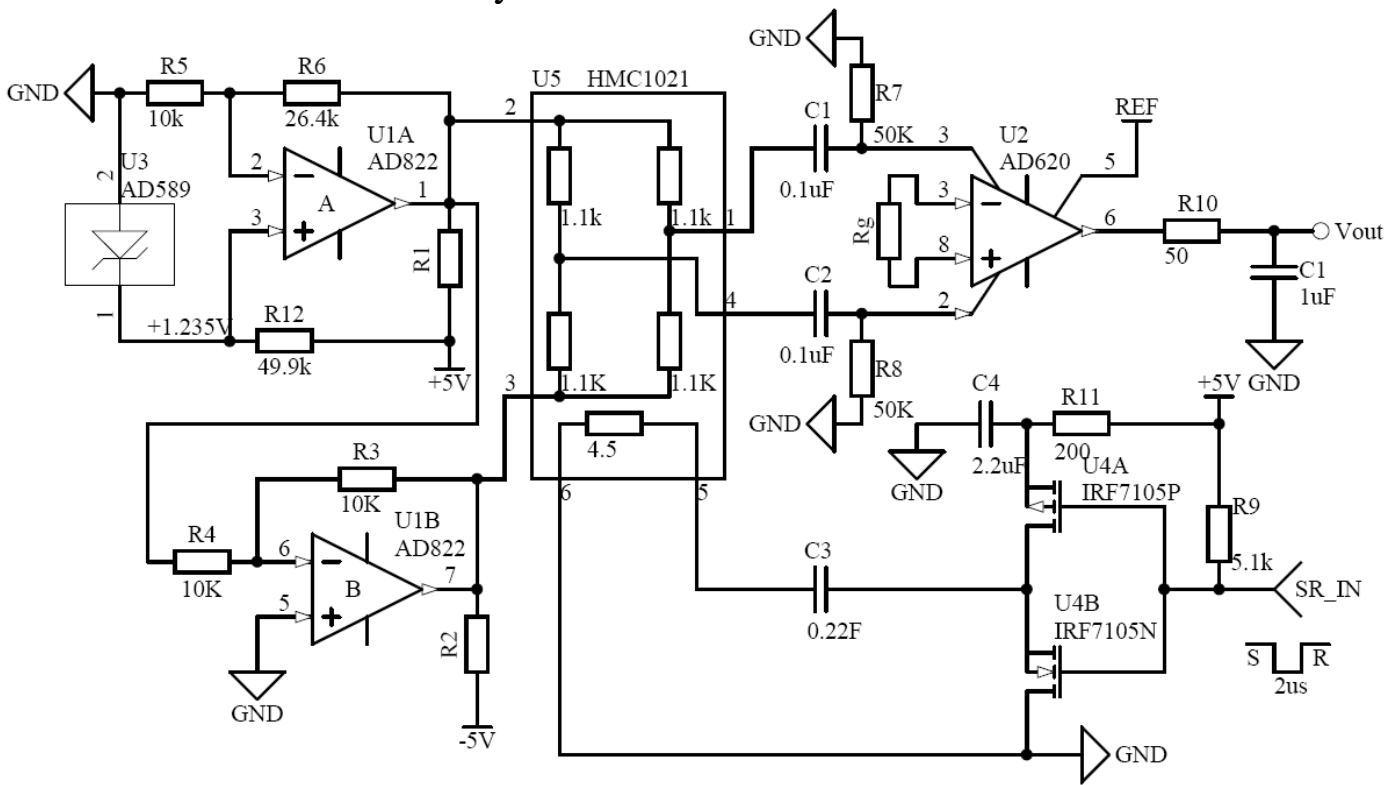

Fig.4 The Analog front-end circuit of the magnetic probe HMC102

Chip AD822 provides HMC1021 bipolar drive current. It can simply be treated as two operational amplifiers, with one output $4.5 \mathrm{~V}$ positive voltage, another as a unity gain inverting amplifier, output $4.5 \mathrm{~V}$ negative voltage. Between them and a negative and positive $5 \mathrm{~V}$ power supply respectively are two resistances. This formates constant current source, which can supply HMC1021 current ,and maintain high sensitivity of the sensor.

Resistance R3 and capacitor C3 form a high pass filter, which can filter out part of the noise, and eliminate the DC offset voltage outputted from the HMC1021. AD620 is a low cost and high precision instrumentation amplifier with low power consumption (1.3mA), low offset voltage (maximum 50mV), low offset drift (maximum $0.6 \mathrm{mV} / \mathrm{C}$ ) characteristics, high common mode rejection ratio, is very suitable for amplification of differential mode signal.And its gain range is from 1 to 1000 , calculated as

$$
\text { Gain }=49.4 \mathrm{k} \Omega / R_{g}+1
$$

Its output is connected to a first-order RC low pass filter, due to the design of the system to detect the 40th harmonic $(2 \mathrm{kHz})$, so the filter's cutoff frequency is about $3 \mathrm{kHz}$.

\section{Experiment}

In order to check the performance of the system designed in the paper, sample a section of data with no signal(noise) with sampling frequency $5 \mathrm{KHz}$, and then obtain the signal(see fig.5) to be analyzed by adding up the noise and a square wave with amplitude $4 / \pi$, frequency $50.2 \mathrm{~Hz}$. The signal to be analyzed will only contain odd number harmonics, and the harmonic amplitude is inversely proportional to the number of harmonics, For example the amplitude of the $2 n+1$ harmonic is $1 /(2 n+1)$.

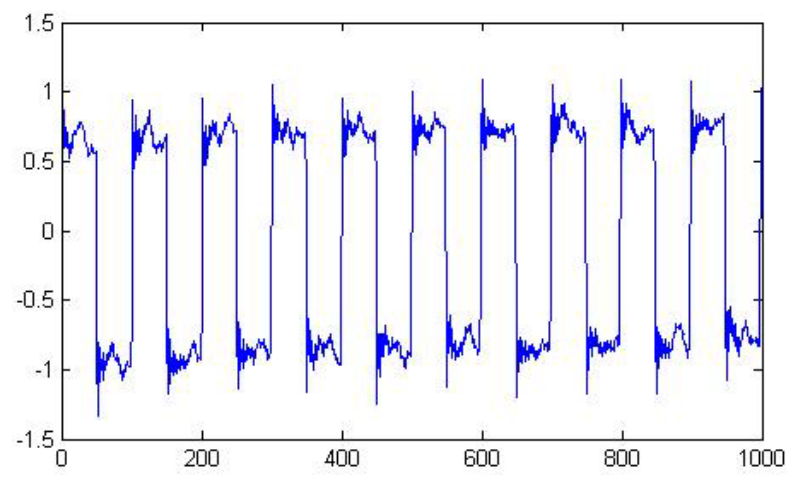

Fig.5 signal to be analyzed 
The final test results are shown in Table 2. The results show that the amplitude and frequency of the first 11 harmonics are close to the true value. The maximum error is $0.1 \mathrm{~Hz}$ and the relative error of amplitude is less than $5 \%$. Thus the system can effectively detect the amplitude and frequency of the power line.

Table 2 experimental results

\begin{tabular}{ccccccc}
\hline Harmonic number & 1 & 3 & 5 & 7 & 9 & 11 \\
\hline Frequency $(\mathrm{Hz})$ & 50.2068 & 150.5508 & 251.0287 & 351.5246 & 451.7875 & 552.1919 \\
Amplitude & 0.9990 & 0.3316 & 0.2031 & 0.1390 & 0.1055 & 0.0904 \\
\hline
\end{tabular}

\section{Conclusion}

In this paper, a motor current harmonic detection system is designed, which makes use of the non contact detection method of the magnetic sensor to detect the harmonic components of the power line, and the FFT algorithm is used to analyze the spectrum of the harmonic components, and the accuracy of the FFT spectrum analysis is overcome. Experimental results show that the system can accurately detect the frequency and amplitude of the harmonics in the power line current signal.

\section{Acknowledgments}

The authors thank Dasha Zhang, Zhuohan Zhang for critical reading of the manuscript; Laboratory of measurement and control technology for providing experimental data for this study.

Communication author is Jian Jiang,the first auther of this paper.Tel:13107210194,Address: College of Mechatronics Engineering and Automation, National University of Defense Technology, Changsha 410073, Hunan, China.

\section{References}

[1]Ma Xing, Li Handong. Research on harmonic detection system based on DSP [J]. Automation and Instrumentation, 2015, (6): 126-132

[2] Hou Liming. A smart grid harmonic detection system architecture of [J]. Wireless Internet Technology, 2014, (5): 63-63

[3] Li Jian, Ma Xin Chun, Tan Zhenzhen, et al. Research of harmonic detection system aased on windowed interpolatation FFT [J]. Electronic design engineering, 2014, 22 (11): 29-31

[4] Cui Xiaorong, Wang Jun, Cao Lin. Current situation and development trend of power system harmonic detection method [J]. electric measurement and instrumentation, 2012,49 (5): 6-10

[5] Zhang Nairao. Neural network and fuzzy control [M]. Beijing: Tsinghua University press, 1998

[6] Tian Wenbo, Yu Jianming, et al. The harmonic parameters of.Dolph-Chebyshev window interpolation [J]. FFT power system and its automation, 2014,26 (2): 50-54

[7] Zhou Xifeng, Zhao Rong, et al..Blackman-Harris window interpolation FFT harmonic analysis and application of [J]. electric measurement and instrumentation, 2014,51 (11): 80-85.

[8] Wen He, Teng Zhaosheng.Hanning self convolution window function and its application of harmonic analysis [J]. China series E: Science and technology, 2009,39 (6): 1190-1198.

[9] Qi Guoqing. Several FFT based frequency estimation methods for precision analysis of [J]. Vibration Engineering Journal, 2006,19 (1): 86-92.

[10] Pan Zhongming. Signal system and control based tutorial [M]. Beijing: Higher Education Press, 2012

[11] Jain V K, Collins W L, Davis DC. High-accurancy analog measurements via interpolated 
FFT[J]. IEEE Trans On Instrum Meas,1979,28(6):113-122.

[12].Grandke T. Interpolation algorithms for discrete Fourier transforms of weighted signals[J]. IEEE Trans On Instrum Meas,1983,32(6):350-355.

[13] Li Xiwen, Zhao Jian, Li Zhiqi et al. Sensor and signal conditioning technology [M]. Xi'an: University of Electronic Science and Technology Press.2008.12:5-8 